\title{
k-NN and k-NN-ANN Combined Classifier to Assess MOX Gas Sensors Performances Affected by Drift Caused by Early Life Aging
}

\author{
Marco Abbatangelo ${ }^{1, *(\mathbb{C})}$, Estefanía Núñez-Carmona ${ }^{2}$ (D) , Veronica Sberveglieri ${ }^{2,3} \mathbb{C D}^{\text {, }}$ \\ Elisabetta Comini ${ }^{1,3}$ (D) and Giorgio Sberveglieri 1,3 \\ 1 Department of Information Engineering, University of Brescia, Brescia, via Branze, 38, 25123 Brescia, Italy; \\ elisabetta.comini@unibs.it (E.C.); giorgio.sberveglieri@nasys.it (G.S.) \\ 2 CNR-IBBR, Institute of Bioscience and Bioresources, via Madonna del Piano, 10, 50019 Sesto Fiorentino, Italy; \\ estefania.nunezcarmona@ibbr.cnr.it (E.N.-C.); veronica.sberveglieri@ibbr.cnr.it (V.S.) \\ 3 Nano Sensor Systems, NASYS Spin-Off University of Brescia, Brescia, via Camillo Brozzoni, \\ 9, 25125 Brescia, Italy \\ * Correspondence: m.abbatangelo@unibs.it
}

Received: 12 November 2019; Accepted: 30 December 2019; Published: 3 January 2020

\begin{abstract}
The drift of metal oxide semiconductor (MOX) chemical sensors is one of the most important topics in this field. The work aims to test the performance of MOX gas sensors over the aging process. Firstly, sensors were tested with ethanol to understand their behavior and response changes. In parallel, beers with different alcoholic content were analyzed to assess what happened in a real application scenario. With ethanol analysis, it was possible to quantify drift of the baseline of the sensors and changes that could affect their responses over time (from day 1 to day 51). Conversely, the beer dataset has been exploited to evaluate how two different classifiers perform the classification task based on the alcohol content of the samples. A hybrid k-nearest neighbors artificial neural network (k-NN-ANN) approach and "standard" k-NN were used to evaluate to distinguish among the samples when the measures were affected by drift. To achieve this goal, data acquired from day one to day six were used as training to predict data collected up to day 51. Overall, performances of the two methods were similar, even if the best result in terms of accuracy is reached by k-NN-ANN $(96.51 \%)$.
\end{abstract}

Keywords: MOX sensors; drift counteraction; aging; beer; k-NN; ANN

\section{Introduction}

Metal oxide semiconductor (MOX) gas sensors are characterized by high sensitivity, fast response, and low cost. Those characteristics make their application very promising in different fields, such as agri-food quality and safety [1-5], environmental monitoring [6-8], home security [9-11], and human health [12-14] among the most investigated. Although these features make MOX sensors one of the most promising technologies of recent years, their diffusion is limited due to some disadvantages. The most crucial is drift, which can change dramatically the information content of the features and cause a reduction of the performance over time.

In the last decade, various methods have been proposed to counteract sensors' drift with good results. Among all those proposed, here some approaches that differ from one another are briefly presented to provide an overview that is as wide as possible. Orthogonal signal correction (OSC) was first applied to counteract gas sensors drift by Padilla, et al. [15]. They showed that sensors discrimination towards three gases at different concentrations was improved if compared to results obtained with non-corrected data. Vergara, et al. used an ensemble of classifiers based on support vector machine (SVM) where each SVM classification output was opportunely weighed [16]. Instead, 
Fornollosa, et al. used a master-slave approach with five twin sensors' arrays for two reasons, but the most interesting was the mitigation of drift effect [17]. With the implementation of a multivariate technique called direct standardization, a multivariate technique that allows mapping an unknown space (in this case, the space from the slave unit) to a reference space (the one from the master unit), they were able to maintain the prediction error stable over time. Finally, Magna, et al. developed a system that improves significantly classification accuracy combining a static feature selection during the training phase and a dynamic feature selection during tests [18].

This study aimed to assess the possibility of using MOX sensors as soon as they are produced, skipping the standard aging step that usually lasts many hours or days, depending on the typology of the sensor [19]. Within the production process, aging is a phase that is induced and controlled to completely oxidize the material and to stabilize it. Hence, the instability for aged sensors is less impactful than for as-fabricated devices [20]. As examples, nanowires made of $\mathrm{CuO}$ may need up to a period of two weeks in ambient air at $300{ }^{\circ} \mathrm{C}$ to complete the aging [21], whereas it has been proved that gallium indium zinc oxide is more stable after being at $250{ }^{\circ} \mathrm{C}$ for $65 \mathrm{~h} \mathrm{[22].}$

This approach wanted to quantify the drift that affects the sensors and the changes in the response for 51 days after their production. During this period, sensors were kept at high temperatures for their correct working. In that way, a sort of aging has been induced in them, but not controlled, since in the same time interval measurements of ethanol and beers have been taken. Four types of sensors were considered and two or three replicas of the same typology of the sensor were used. These sensors were placed inside an S3 (small sensor systems), an e-nose-like device, to exploit its advantages: automatic execution of samples under analysis (once set) and transmission of data to a dedicated web app.

The datasets thus obtained were used to assess two machine learning algorithms regarding the ability to counteract drift in the "early life" of sensors and to assure reliable performances over time. On one hand, measures with ethanol have been elaborated to evaluate drift and changes in the sensors' responses. On the other hand, a k-nearest neighbors (k-NN) approach has been used alongside a hybrid method that combines k-NN with artificial neural networks (ANN) for the "beer dataset". The aim was to assess if the application of one of the most used computational systems, i.e., ANN, could enhance overall performances. To the best of our knowledge, such a machine learning approach has not been applied to chemical sensors, although it has been shown to yield promising results in other fields, such as classification of clouds through images [23] or disease like diabetes and cancer [24]; finally, it differs from the method proposed by [25], i.e., an ensemble of k-NN and ANN classifiers used to counteract sensors drift.

\section{Materials and Methods}

\subsection{S3 Device}

S3 is a device developed by the collaboration of Sensor Lab at the University of Brescia and Nasys S.r.l., a spin-off of the same University. S3 was previously used in numerous studies with considerable success; particularly, it has been applied to the field of food technology and quality control [26-28]. S3 consists of an electronic part to manage the signal, an element that allows its connection to the internet, a pump to bring the volatile compounds to the heart of the instrument from an autosampler HT2010H (HTA s.r.l., Brescia, Italy). It supports a 42-loading site carousel that can reduce the possible variables due to the preparation of the sample.

The sensors grown and developed for this work were created with two techniques, rheotaxial growth thermal oxidation (RTGO) and nanowire technology. The RGTO technique involves two deposition steps: the first stage of a metallic thin film by DC magnetron sputtering from a metallic target on a substrate at higher temperatures than the melting point of the metal, then the thermal oxidation cycle to get a metal oxide layer with stable stoichiometry [29]. In this work, tin oxide RGTO and tin oxide RGTO functionalized with gold particles were put in the chamber of S3. Nanowires (NW) exhibit exceptional crystalline quality and a very high length-to-width ratio, subsequent in enhanced 
sensitivity as well as long-term material stability for prolonged operation [30,31]. The experimental process consists of the evaporation of the powder (metal oxide) at high temperatures in a controlled atmosphere at pressures lower than hundreds of millibar and the following mass transport of the vapor towards the substrates kept at lower temperatures concerning the source evaporation region. The list of the 10 sensors produced at Sensor Lab and used for this study are presented in Table 1.

Table 1. List of the ten sensors used in the study.

\begin{tabular}{cccc}
\hline Material (Type) & Number of Replicas & Morphology & Working Temperature $\left({ }^{\circ} \mathbf{C}\right)$ \\
\hline $\mathrm{SnO}_{2} \mathrm{Au}(\mathrm{n})$ & 3 sensors & RGTO & 450 \\
$\mathrm{SnO}_{2}(\mathrm{n})$ & 3 sensors & RGTO & 450 \\
$\mathrm{SnO}_{2} \mathrm{Au}(\mathrm{n})$ & 2 sensors & Nanowire & 350 \\
$\mathrm{CuO}(\mathrm{p})$ & 2 sensors & Nanowire & 350 \\
\hline
\end{tabular}

\subsection{Experimental Set-Up}

Eight sessions of measures were realized within 51 days. The first session has been done before any aging of the sensors; other sessions were carried out at the following days: 2, 6, 8, 26, 30, 37, and 51. After the first measure, sensors were kept at their operating temperature throughout the measurement period. Two different tests were designed for each day of measure, waiting a period of $1 \mathrm{~h}$ among them. The first test was made with 300 ppm of ethanol (dry air), repeating the measures 10 times each day; the second one with four different beers. Beers differed for the alcohol content: two were nonalcoholic beers, having $0.0 \%$ and $0.5 \%$ of alcohol by volume (ABV); the other two had an ABV of $4.7 \%$ and $7.9 \%$. The choice to analyze this type of product is mainly due to understanding the response of the sensors in a possible application scenario, bearing in mind that ethanol is one of the most volatile compounds that characterize it. Proceeding in this way, it was possible to make a comparison between the two tests. Every single sample was analyzed for $15 \mathrm{~min}$ : sensors were exposed to ethanol and beer VOCs for $2 \mathrm{~min}$; $13 \mathrm{~min}$ were necessary to restore the baseline with air.

\subsection{Data Analysis}

The measures obtained in the 8 days have been divided to form two different datasets. The first one contained the analysis carried out using ethanol. From this dataset, two kins of information have been extrapolated: how sensors drifted and how much their response changes in the first 51 days after their production. As an indicator of the response, the difference between the first value of the acquired resistance $\left(R_{0}\right)$ and its minimum value (for $n$-type sensors) or the maximum value (p-type sensors) during the analysis time was calculated; the difference was divided by $R_{0}$ to get the percentage variation respect to the baseline. Hence, $\Delta R / R_{0}$ has been extracted as a feature for the following analysis.

The second dataset ("beer dataset") included an analysis of the four beer samples during the same period. In this case, the effect of drift in classification tasks has been evaluated. Principal component analysis (PCA) was used as an unsupervised technique to visualize data, reduce dimensionality and assess if the drift affected the four samples in the same way. From the PCA score plot, it has been decided to use the k-NN algorithm to obtain the accuracy of the system, using Euclidean distance as a metric. The possibility to use a hybrid algorithm based on k-NN was also investigated to try to enhance classification performances. In particular, a method similar to the one described in [23] has been used. The main difference stands in the use of "scaled conjugated gradient" as a learning algorithm instead of "extreme learning machine"; furthermore, different architectures of ANN were investigated to find the best one in terms of classification performances. This approach consists of 5 steps.

1. ANNs were trained using data from day 1 to day 6. Six different ANNs were tested: two of them had 1 hidden layer of 10 neurons and differed for the activation function, i.e., ReLu and 
hyperbolic tangent sigmoid; the other four networks had 2 layers of 10 and 7 neurons and a combination of the abovementioned activation functions was used.

2. For the jth sample of the test set, the distance from the samples of the training set was calculated using Euclidean distance.

3. k-nearest neighbors were chosen; then the input for the ANN was calculated with the following formula:

$$
\text { Input }=\left[\sum_{i=1}^{k} \frac{F_{i}^{1}}{k}, \sum_{i=1}^{k} \frac{F_{i}^{2}}{k}, \ldots, \sum_{i=1}^{k} \frac{F_{i}^{n}}{k}\right]
$$

where $F^{1}, F^{2}, \ldots, F^{n}$ are the features that describe samples. Hence, the input of the network is a vector that has as features the mean value of the same parameters of the k-nearest neighbors.

4. ANNs were applied and the class of the sample was predicted.

5. Steps from 1 to 4 were repeated for all samples in the test set.

Data analysis has been performed with Matlab ${ }^{\circledR}$ R2015a software (MathWorks, Natick, MA, USA) and Python.

\section{Results and Discussion}

In Figure 1 sensors' outputs at 300 ppm of ethanol for each typology of sensor are reported. One sensor for each type has been chosen; in particular, those that will be named as "sensor \#1" from here on. For each day, the plotted signal represents the mean value of the ten measures done in that day. The two phases of the measure are clearly portrayed: during the first one, sensors are exposed to gas and hence their resistance decreases for n-type $\left(\mathrm{SnO}_{2}\right)$ and increases for p-type $(\mathrm{CuO})$; in the second phase, pure air is fluxed into sensor and the baseline is restored.
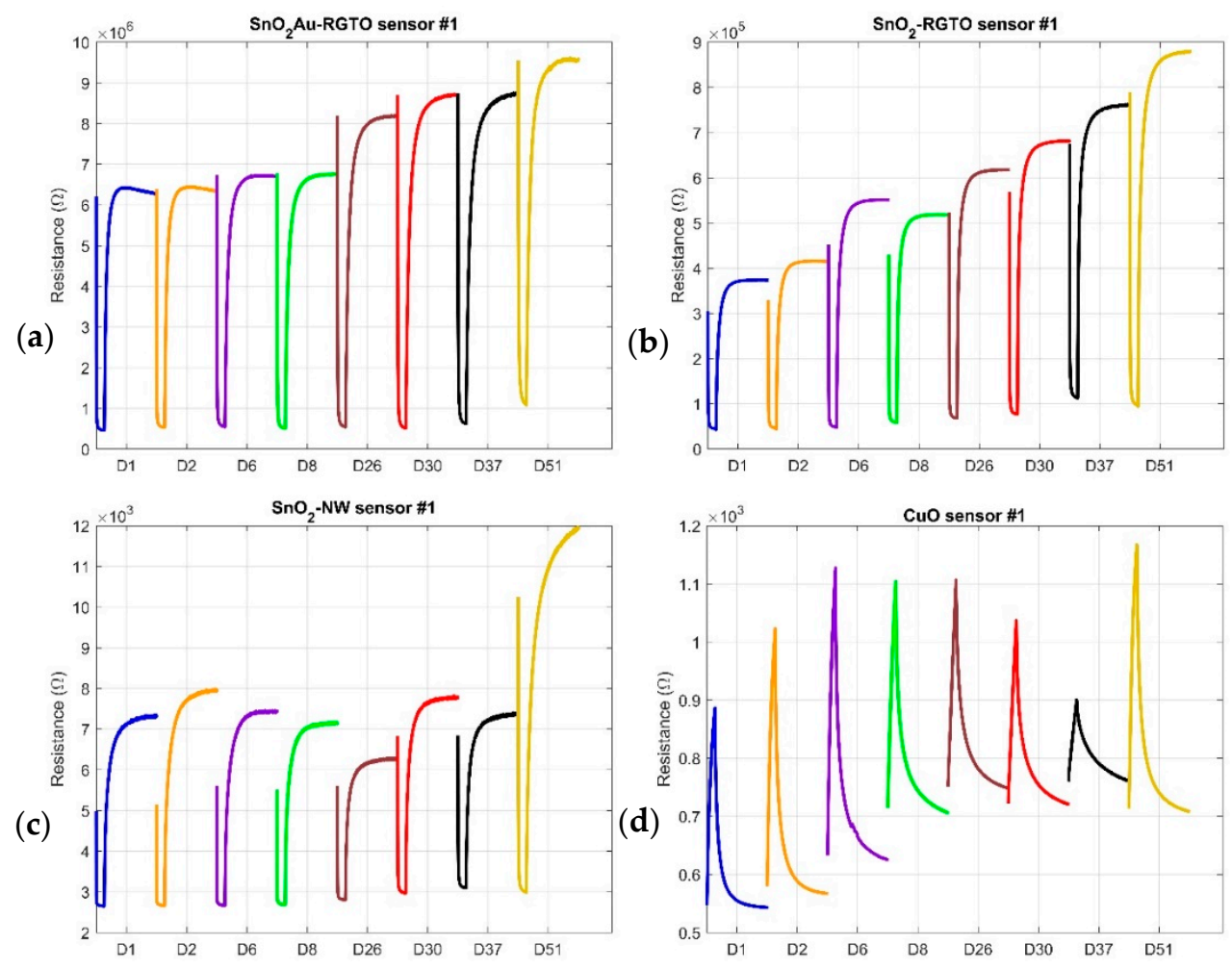

Figure 1. Outputs from all sensors' typologies to $300 \mathrm{ppm}$ of ethanol, from day 1 to day 51 . Each signal is the mean of the ten signals acquired the same day. (a) $\mathrm{SnO}_{2} \mathrm{Au}$ RGTO sensor \#1; (b) $\mathrm{SnO}_{2} \mathrm{RGTO}$ sensor \#1; (c) $\mathrm{SnO}_{2} \mathrm{NW}$ sensor \#1; (d) CuO sensor \#1. 
Figure 2 shows the trend of baseline from day 1 to day 51 for all the sensors, divided for typology. Results are presented in terms of mean value, with the error bars representing the standard deviation. Sensors of the same material and morphology are grouped to compare their behavior. Regarding $\mathrm{SnO}_{2} \mathrm{Au}$ RGTO sensors, two of them had a stable baseline over time, whereas the third one it started increasing after 8 days and reached a mean value at day 51 that is $53.67 \%$ higher respect to the initial value. On the contrary, $\mathrm{SnO}_{2}$ RGTO sensors were all affected by a drift that caused an increase of the baseline at day 51 of 2.58 times for sensor \#1 to 2.76 times for the other two respect to the value of the day 1.

(a)

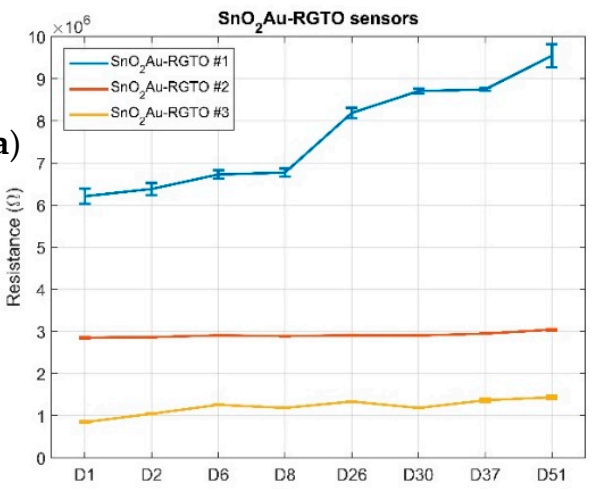

(c)

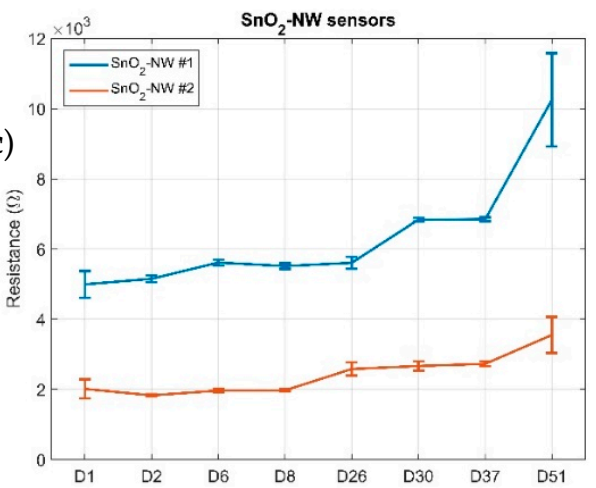

(b)
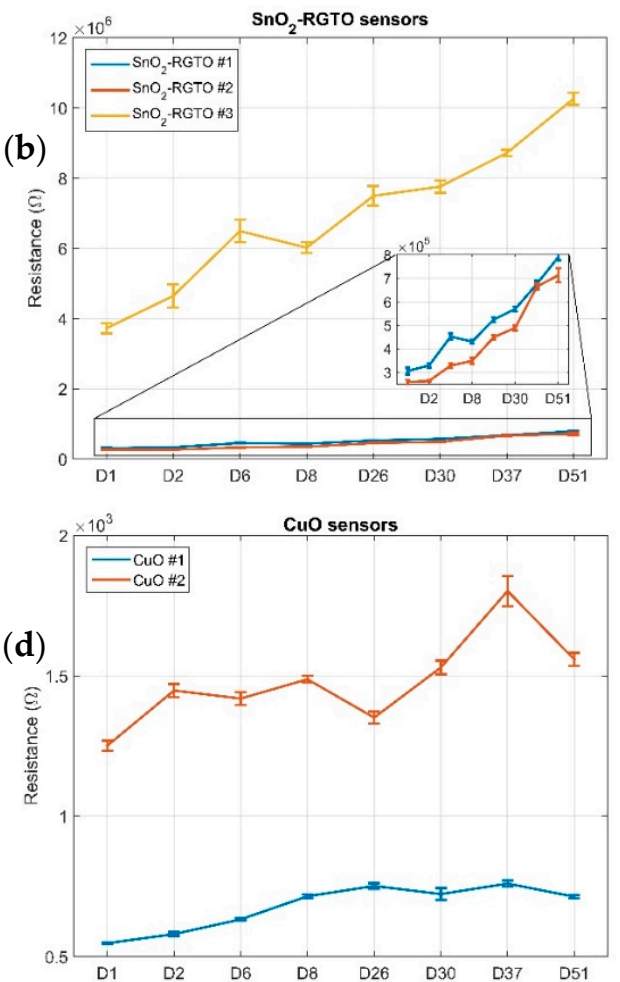

Figure 2. Baseline drift of all the sensors from day 1 to day 51, divided for typology. (a) Drift of the 3 $\mathrm{SnO}_{2} \mathrm{Au}$ RGTO sensors; (b) drift of the $3 \mathrm{SnO}_{2}$ RGTO sensors; the trend of sensor \#1 and \#2 is zoomed in the box on the right part of the image; (c) drift of the $2 \mathrm{SnO}_{2} \mathrm{NW}$ sensors; (d) drift of the $2 \mathrm{CuO}$ sensors.

Best results have been obtained with NW sensors. Indeed, the ones made of $\mathrm{CuO}$ had a resistance value in air that increased between $30.28 \%$ and $24.55 \%$ during the observation period, while the growth for tin oxide NWs between $105.37 \%$ and $76.44 \%$.

In Figure $3, \Delta R / R_{0}$ is reported. These values were taken as indicators of the responses of the sensors. From the data obtained, it results that both typologies of RTGO sensors have a response that is quite stable for 51 days, oscillating between 0.88 and 0.96 for $\mathrm{SnO}_{2} \mathrm{Au}$ and between 0.81 and 0.89 for $\mathrm{SnO}_{2}$. Regarding $\mathrm{SnO}_{2} \mathrm{Au}$ RGTO, sensor \#2 has not been included in Figure 3, but its response was lower than 0.08 for this type of measure. Conversely, NW sensors have different trends. Tin oxide NWs are characterized by an increase of their resistance variation in day 51 in respect to day 1 , and fluctuation of the same parameter up to day 37. Finally, $\mathrm{CuO}$ sensor \#2 has a behavior more similar to RGTO in terms of reproducibility, whilst the same feature for $\mathrm{CuO}$ sensor \#1 has an oscillating trend from 0.18 to 0.78 , that cannot be considered a linear drift if compared with the other sensors of the array. 

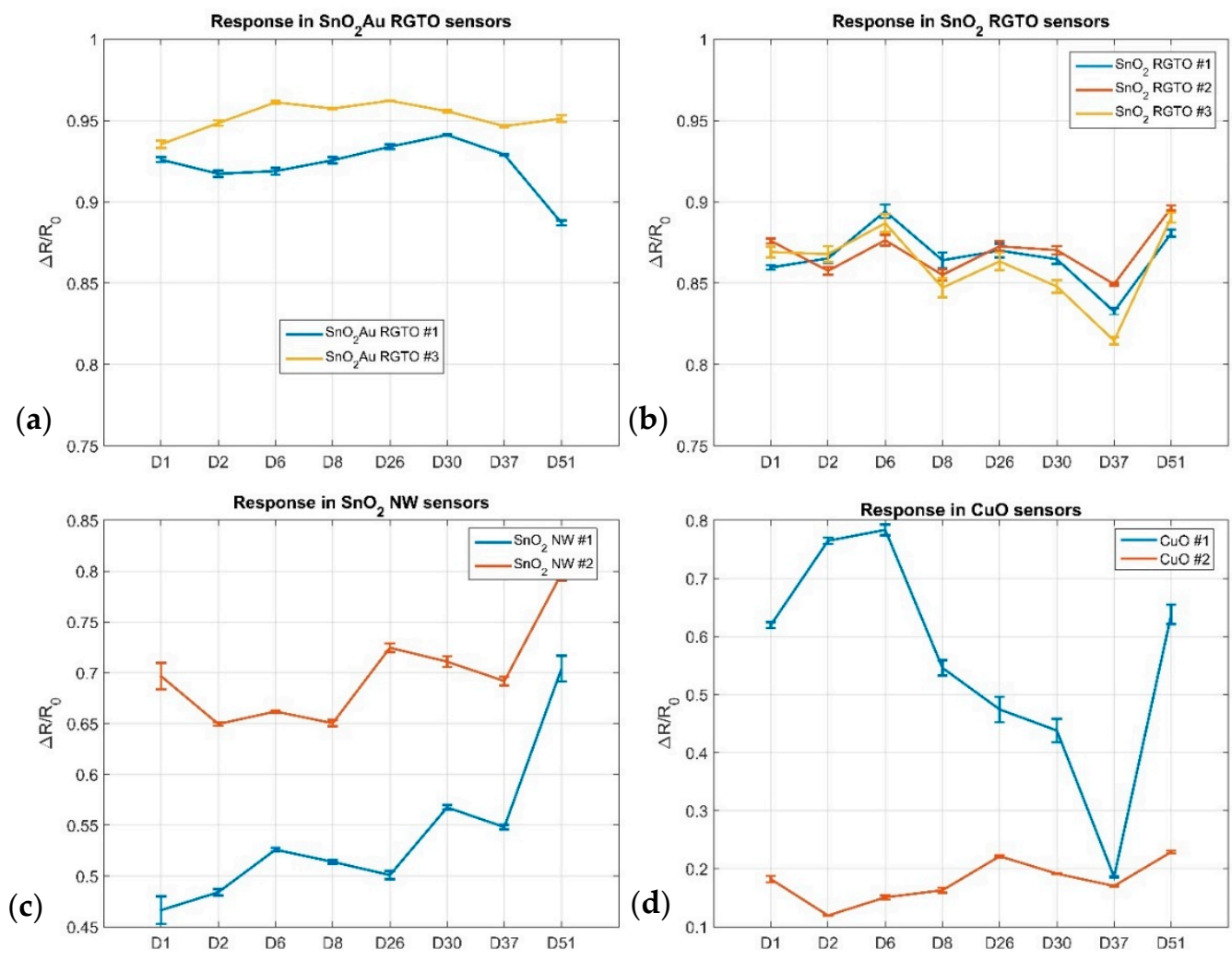

Figure 3. $\Delta R / R_{0}$ for all the sensors from day 1 to day 51. (a) Response of 2 out of $3 \mathrm{SnO}_{2} \mathrm{Au} \mathrm{RGTO}$ sensors; (b) response of the $3 \mathrm{SnO}_{2} \mathrm{RGTO}$ sensors; (c) response of the $2 \mathrm{SnO}_{2} \mathrm{NW}$ sensors; (d) response of the $2 \mathrm{CuO}$ sensors.

For the "beer dataset", PCA was performed as explorative analysis to visualize the drift that affected beer measures. In Figure 4, the PCA score plot of the first two principal components (PCs) is shown. The total explained variance (EV) is equal to $90.5 \%$ ( $87.08 \%$ for PC1 and $8.82 \%$ for PC2), meaning that most of the information is contained in two variables. A clear distinction between the four beers classes is achieved considering samples scores along PC1. However, the distance among the clusters tends to decrease as the ABV increases. This result could be explained by the fact that alcohol amount of $4.7 \%$ and $7.9 \%$ beers brings sensors near to saturation, making the distinction between these two classes less clear. On the contrary, on the PC2 axis drift can be seen. Additionally, in this case, different considerations can be done for the two lower and the two higher ABV beers. The entity of the dispersion for $0.0 \%$ and $0.5 \%$ samples is weak. Nevertheless, a separation between data acquired the first 6 days and those obtained from day 26 onwards can be observed. For the other two classes, the day-to-day difference is more pronounced within the same class. The same observation regarding measures before and after day six is valid in this case, too. The main cause of this result is due to the drastic change in $\mathrm{CuO} \# 1$ sensor response that drops from the maximum value of 0.78 to 0.54 . The subsequent discontinuous behavior is mitigated by the stable response recorded from the other sensors in the array, especially RGTO ones.

From PCA results, the k-NN algorithm seemed to be a good candidate to evaluate the classification performance of the sensors. It has been decided to use data from the first six days as training set (45 samples) and data from day 26 to day 51 as test set (86 samples). The aim was to assess the correct classification of the system when trained only with few initial patterns and forced to recognize patterns temporally distant belonging to the same classes. Hence, the intent was to verify whether the methods proposed succeeded in reducing the negative effect due to sensor drift. Side by side with k-NN, a hybrid algorithm that combines k-NN and ANNs has been investigated. The main target was to 
exploit the non-linearity of the ANNs to improve the performance of k-NN. Both the approaches were tested varying the number of $k$ neighbors from 1 to 15 .

From PCA, a subset of 3 features has been chosen to optimize the classification task. In detail, $\mathrm{SnO}_{2} \mathrm{Au}$ RGTO \#1 and the two $\mathrm{SnO}_{2} \mathrm{NW}$ sensors have been selected. Percentages of accuracy are shown in Figure 5. k-NN performances have been highlighted with a thicker and dashed line to better understand if the ones of the hybrid method could get over them.

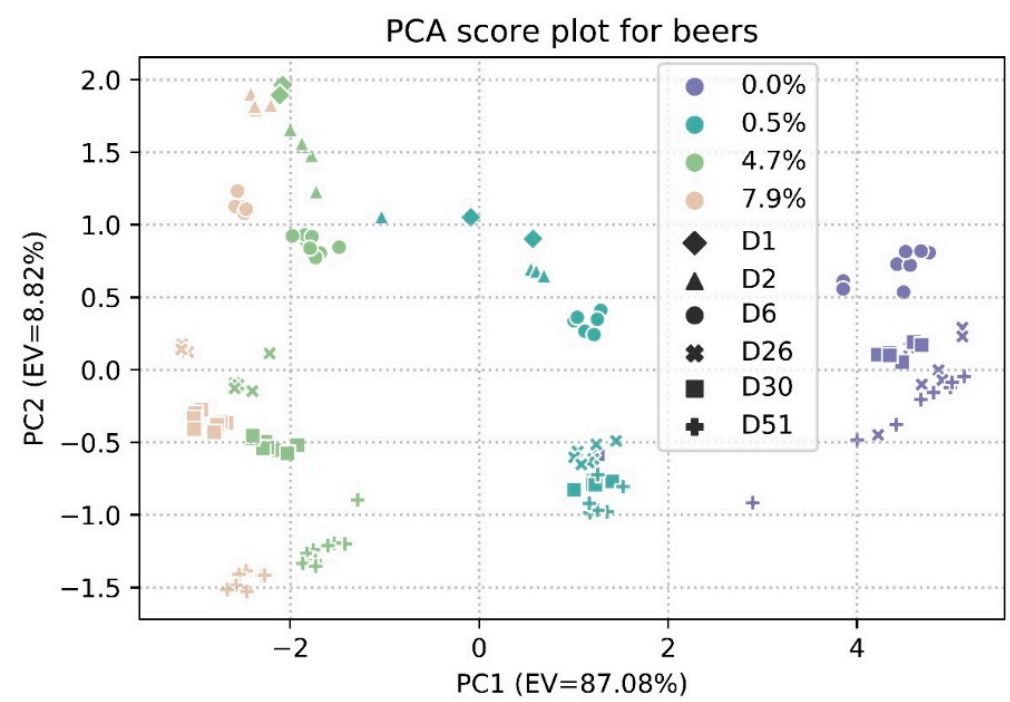

Figure 4. PCA score plot for beer samples. Total explained variance (EV) equal to $95.9 \%$ from the first two principal components (PC1 EV $=87.08 \%$, PC2 EV $=8.82 \%$ ). Colors indicate the different ABV of the samples, markers denote different days (from 1 to 51 ).

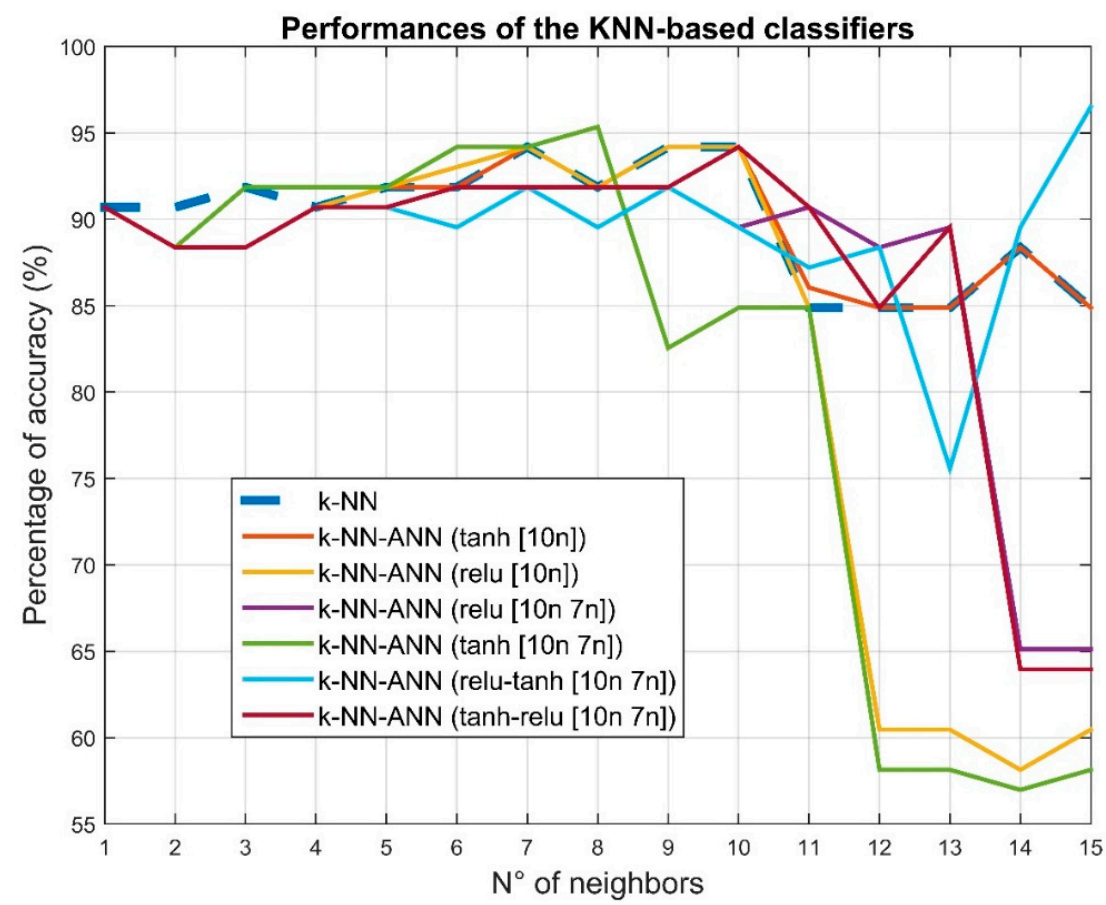

Figure 5. Accuracies of k-NN and the different ANNs structures for the combined method. Among square brackets the number of neurons per layer is indicated; tanh denotes the hyperbolic tangent sigmoid activation function, relu is rectified linear unit function.

k-NN-ANN accuracies are consistent with those obtained with k-NN since there is no significant difference between the different percentages. However, with the increasing number of neighbors, 
the combined method reduces performances in some architectures, especially those with ReLu in the last hidden layer. This reduction of performance is caused by the misclassification among the samples of classes of $4.7 \%$ and $7.9 \% \mathrm{ABV}$ beers. It can be assumed that the class boundaries learned by the ANN were degraded when $k$ becomes higher: indeed, in this case, almost samples of the two classes used in training are considered as a reference for the calculation of the distance and ReLu function is not able to learn correctly. The only exception is represented by the ANN with ReLu and hyperbolic tangent sigmoid as activation functions of the first and the second layer respectively (cyan line). In this case, the best result is obtained with $k=15$ reaching a value of $96.51 \%$, which is also the highest accuracy achieved. Generally, it possible to affirm that both methods are able to learn the classes of the sample using a small part of the dataset and to assign the measure used as test to the correct belonging class, thus limiting the negative effect of drift.

\section{Conclusions}

This study aimed to characterize how sensors, just produced and without induced aging, drift and how it is possible to counteract it. Ten different sensors divided into four groups were tested (RGTO and NW, tin oxide and copper oxide) with ethanol and beers with different alcohol content. Two parameters were considered: baseline drift and changes in resistance variation when exposed to gases. From ethanol measures, it resulted that NW sensors (both tin and copper oxide) had the most stable baseline during the period of analysis (days 1-51). Conversely, RGTO sensors showed more reproducibility over time.

One hybrid approach that combines k-NN and ANNs has been used to evaluate the possibility to counteract drift, using the dataset containing beers measures. Performances were compared with the k-NN algorithm. It resulted that the two approaches do not differ from each other in terms of accuracy, although the best classification result was achieved with the hybrid method.

Author Contributions: Conceptualization, V.S., E.C., and G.S.; methodology, E.N.-C., and V.S.; software, M.A.; validation, M.A., E.N.-C., V.S., E.C., and G.S.; formal analysis, M.A.; investigation, M.A., E.N.-C., and V.S.; resources, E.C. and G.S.; data curation, M.A.; writing-original draft preparation, M.A.; writing-review and editing, E.N.-C, V.S., E.C., and G.S.; supervision, E.C. and G.S. All authors have read and agreed to the published version of the manuscript.

Funding: This research received no external funding.

Conflicts of Interest: The authors declare no conflict of interest.

\section{References}

1. Xu, M.; Ye, L.; Wang, J.; Wei, Z.; Cheng, S. Quality tracing of peanuts using an array of metal-oxide based gas sensors combined with chemometrics methods. Postharvest Biol. Technol. 2017, 128, 98-104. [CrossRef]

2. Gliszczyńska-Świgło, A.; Chmielewski, J. Electronic Nose as a Tool for Monitoring the Authenticity of Food. A Review. Food Anal. Method 2017, 10, 1800-1816. [CrossRef]

3. Peris, M.; Escuder-Gilabert, L. Electronic noses and tongues to assess food authenticity and adulteration. Trends Food Sci. Technol. 2016, 58, 40-54. [CrossRef]

4. Gancarz, M.; Wawrzyniak, J.; Gawrysiak-Witulska, M.; Wiącek, D.; Nawrocka, A.; Tadla, M.; Rusinek, R. Application of electronic nose with MOS sensors to prediction of rapeseed quality. Measurement 2017, 103, 227-234. [CrossRef]

5. Galstyan, V.; Bhandari, M.P.; Sberveglieri, V.; Sberveglieri, G.; Comini, E. Metal Oxide Nanostructures in Food Applications: Quality Control and Packaging. Chemosensors 2018, 6, 16. [CrossRef]

6. Fabbri, B.; Valt, M.; Parretta, C.; Gherardi, S.; Gaiardo, A.; Malagù, C.; Mantovani, F.; Strati, V.; Guidi, V. Correlation of gaseous emissions to water stress in tomato and maize crops: From field to laboratory and back. Sens. Actuators B Chem. 2020, 303, 127227. [CrossRef]

7. Bilgera, C.; Yamamoto, A.; Sawano, M.; Matsukura, H.; Ishida, H. Application of Convolutional Long Short-Term Memory Neural Networks to Signals Collected from a Sensor Network for Autonomous Gas Source Localization in Outdoor Environments. Sensors 2018, 18, 4484. [CrossRef] [PubMed] 
8. Vuka, M.; Schaffernicht, E.; Schmuker, M.; Bennetts, V.H.; Amigoni, F.; Lilienthal, A.J. Exploration and localization of a gas source with MOX gas sensors on a mobile robot-A Gaussian regression bout amplitude approach. In ISOCS/IEEE International Symposium on Olfaction and Electronic Nose (ISOEN); IEEE: Piscataway, NJ, USA, 2017; pp. 1-3. [CrossRef]

9. Spinelle, L.; Gerboles, M.; Kok, G.; Persijn, S.; Sauerwald, T. Review of Portable and Low-Cost Sensors for the Ambient Air Monitoring of Benzene and Other Volatile Organic Compounds. Sensors 2017, $17,1520$. [CrossRef]

10. Masson, N.; Piedrahita, R.; Hannigan, M. Approach for quantification of metal oxide type semiconductor gas sensors used for ambient air quality monitoring. Sens. Actuators B Chem. 2015, 208, 339-345. [CrossRef]

11. Herberger, S.; Herold, M.; Ulmer, H.; Burdack-Freitag, A.; Mayer, F. Detection of human effluents by a MOS gas sensor in correlation to VOC quantification by GC/MS. Build. Environ. 2010, 45, 2430-2439. [CrossRef]

12. Sberveglieri, V.; Núñez-Carmona, E.; Ponzoni, A.; Comini, E.; Galstyan, V.; Zappa, D.; Pulvirenti, A. Skin Microbiota Monitoring by Nanowire MOS Sensors. Procedia Eng. 2015, 120, 756-759. [CrossRef]

13. Jaeschke, C.; Gonzalez, O.; Padilla, M.; Richardson, K.; Glockler, J.; Mitrovics, J.; Mizaikoff, B. A Novel Modular System for Breath Analysis Using Temperature Modulated MOX Sensors. Proceedings 2019, 14, 49. [CrossRef]

14. Lawson, B.; Aguir, K.; Fiorido, T.; Martini-Laithier, V.; Bouchakour, R.; Burtey, S.; Reynard-Carette, C.; Bendahan, M. Skin alcohol perspiration measurements using MOX sensors. Sens. Actuators B Chem. 2019, 280, 306-312. [CrossRef]

15. Padilla, M.; Perera, A.; Montoliu, I.; Chaudry, A.; Persaud, K.; Marco, S. Drift compensation of gas sensor array data by Orthogonal Signal Correction. Chemom. Intell. Lab. 2010, 100, 28-35. [CrossRef]

16. Vergara, A.; Vembu, S.; Ayhan, T.; Ryan, M.A.; Homer, M.L.; Huerta, R. Chemical gas sensor drift compensation using classifier ensembles. Sens. Actuators B Chem. 2012, 166-167, 320-329. [CrossRef]

17. Fonollosa, J.; Fernández, L.; Gutiérrez-Gálvez, A.; Huerta, R.; Marco, S. Calibration transfer and drift counteraction in chemical sensor arrays using Direct Standardization. Sens. Actuators B Chem. 2016, 236, 1044-1053. [CrossRef]

18. Magna, G.; Mosciano, F.; Martinelli, E.; Di Natale, C. Unsupervised On-Line Selection of Training Features for a robust classification with drifting and faulty gas sensors. Sens. Actuators B Chem. 2018, 258, 1242-1251. [CrossRef]

19. Adamyan, A.Z.; Adamyan, Z.N.; Aroutiounian, V.M.; Schierbaum, K.D.; Han, S.D. Improvement and stabilization of thin-film hydrogen sensors parameters. Armen. J. Phys. 2009, 2, 200-212.

20. Korotcenkov, G.; Cho, B.K. Instability of metal oxide-based conductometric gas sensors and approaches to stability improvement (short survey). Sens. Actuators B Chem. 2011, 156, 527-538. [CrossRef]

21. Mazhar, M.E.; Faglia, G.; Comin, E.; Zappa, D.; Baratto, C.; Sberveglieri, G. Kelvin probe as an effective tool to develop sensitive p-type CuO gas sensors. Sens. Actuators B Chem. 2016, 222, 1257-1263. [CrossRef]

22. Jung, J.S.; Son, K.S.; Kim, T.S.; Ryu, M.K.; Park, K.B.; Yoo, B.W.; Kwon, J.Y.; Lee, S.Y.; Kim, J.M. Stability Improvement of Gallium Indium Zinc Oxide Thin Film Transistors by Post-Thermal Annealing. ECS Trans. 2008, 16, 309-313. [CrossRef]

23. Xia, M.; Lu, W.; Yang, J.; Ma, Y.; Yao, W.; Zheng, Z. A hybrid method based on extreme learning machine and k-nearest neighbor for cloud classification of ground-based visible cloud imag. Neurocomputing 2015, 160, 238-249. [CrossRef]

24. Le, L.; Xie, Y.; Raghavan, V.V. Deep Similarity-Enhanced K Nearest Neighbors. In Proceedings of the IEEE International Conference on Big Data (Big Data), Seattle, WA, USA, 10-13 December 2018; IEEE: Piscataway, NJ, USA, 2018. [CrossRef]

25. Adhikari, S.; Saha, S. Multiple Classifier Combination Technique for Sensor Drift Compensation using ANN \& KNN. In Proceedings of the IEEE International Advance Computing Conference (IACC), Gurgaon, India, 21-22 February 2014; IEEE: Piscataway, NJ, USA, 2014. [CrossRef]

26. Abbatangelo, M.; Núñez-Carmona, E.; Sberveglieri, V.; Zappa, D.; Comini, E.; Sberveglieri, G. Application of a Novel S3 Nanowire Gas Sensor Device in Parallel with GC-MS for the Identification of Rind Percentage of Grated Parmigiano Reggiano. Sensors 2018, 18, 1617. [CrossRef] [PubMed]

27. Abbatangelo, M.; Núñez-Carmona, E.; Duina, G.; Sberveglieri, V. Multidisciplinary Approach to Characterizing the Fingerprint of Italian EVOO. Molecules 2019, 24, 1457. [CrossRef] [PubMed] 
28. Núñez-Carmona, E.; Abbatangelo, M.; Sberveglieri, V. Innovative Sensor Approach to Follow Campylobacter jejuni Development. Biosensors 2019, 9, 8. [CrossRef] [PubMed]

29. Sberveglieri, G. Recent developments in semiconducting thin-film gas sensors. Sens. Actuators B Chem. 1995, 23, 103-109. [CrossRef]

30. Comini, E.; Faglia, G.; Sberveglieri, G.; Pan, Z. Stable and highly sensitive gas sensors based on semiconducting oxide Nanobelts. Appl. Phys. Lett. 2002, 81, 1869-1871. [CrossRef]

31. Sberveglieri, G.; Concina, I.; Comini, E.; Falasconi, M.; Ferroni, M.; Sberveglieri, V. Synthesis and integration of tin oxide nanowires into an electronic nose. Vacuum 2012, 86, 532-535. [CrossRef]

(C) 2020 by the authors. Licensee MDPI, Basel, Switzerland. This article is an open access article distributed under the terms and conditions of the Creative Commons Attribution (CC BY) license (http://creativecommons.org/licenses/by/4.0/). 